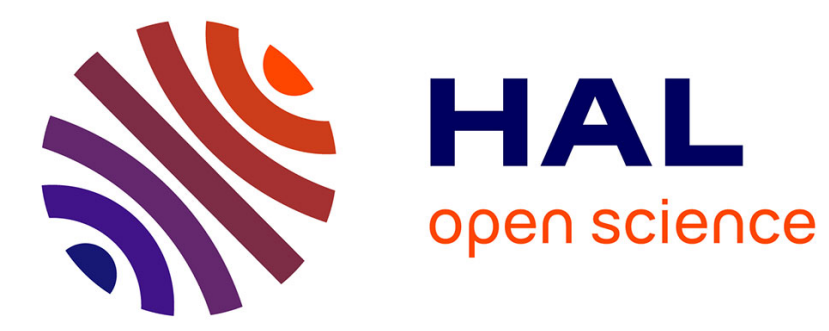

\title{
LOCAL ORDER AND NON EQUILIBRIUM BEHAVIOUR IN GLASSY CRYSTAL CYANOADAMANTANE
}

Michel Descamps, C. Caucheteux, G. Odou

\section{- To cite this version:}

Michel Descamps, C. Caucheteux, G. Odou. LOCAL ORDER AND NON EQUILIBRIUM BEHAVIOUR IN GLASSY CRYSTAL CYANOADAMANTANE. Journal de Physique Colloques, 1985, 46 (C8), pp.C8-329-C8-333. 10.1051/jphyscol:1985849 . jpa-00225192

\section{HAL Id: jpa-00225192 https://hal.science/jpa-00225192}

Submitted on 1 Jan 1985

HAL is a multi-disciplinary open access archive for the deposit and dissemination of scientific research documents, whether they are published or not. The documents may come from teaching and research institutions in France or abroad, or from public or private research centers.
L'archive ouverte pluridisciplinaire HAL, est destinée au dépôt et à la diffusion de documents scientifiques de niveau recherche, publiés ou non, émanant des établissements d'enseignement et de recherche français ou étrangers, des laboratoires publics ou privés. 


\title{
LOCAL ORDER AND NON EQUILIBRIUM BEHAVIOUR IN GLASSY CRYSTAL CYANOADAMANTANE
}

M. Descamps, C. Caucheteux and G. Odou Laboratoire de Dynamique des Cristaux Moléculaires, (U.A. CNRS 801),
Université de Lilize I, 59655 Vilieneuve d'Asca Cedex, France

\begin{abstract}
Résumé - Lors de recuits isothermes en phase plastique trempée, le cyanoadamantane évolue vers une phase métastable dont la structure est différente de celle de la phase stable à la même température. Cette microstructure est décrite. La cinétique de mise en ordre qui est corrélée à une contraction valumique est analysée en terme de relaxation moléculaire.
\end{abstract}

Abstract - During isothermal annealing in the glassy crystal phase, cyanoadamantane relaxes towards a metastable state. The microstructure of the latter is described. It is different from that of the stable phase at the same temperature. The kinetics of ordering which are coupled to a volume contraction are analyzed in terms of molecular relaxation.

The "glassy crystals" /1/ which are obtained by quenching the orientationally disordered phase of some molecular crystals [plastic crystals] are very attractive models of the glassy state as their thermal behaviour reveals the typical features of a classical glass transition whereas the frozen disorder is mainly orientational. Such a behaviour has recently been suspected in cyanoadamantane [CN-ADM] which deserves special attention on account of its simple structure $/ 2 /$.

When CN-ADM is rapialy cooled from the room temperature f-c-c phase [I] to a low enough temperature, the first order transition to the stable antiferro ordered monoclinic phase [II] is avoided $\left[T_{C} \simeq 283 \mathrm{~K}\right.$ at thermal equilibrium]. While reheating, a heat capacity jump which could indicate a glassy transition is observed at about $T_{g}=170 \mathrm{~K}$. The macroscopic crystal symmețry as well as the time averaged structure remain unchanged : the dipolar axis can randomly take six orientations closely located along the $\langle 100\rangle$ directions. But NMR and dielectric experiments / $3 /$ suggest a freezing of this tumbling while the fast uniaxial reorientation is almost unchanged at Tg. Besides, low temperature experiments have revealed characteristic anomalies of amorphous solids [two level systems] $/ 4 /$. We have recently pointed out that annealings of plastic single crystals quenched down in the 150-170 $\mathrm{K}$ range, show slow growing of a Jocal order /5,6\%. It is characterized by X-ray superstructure spots at the $X$-boundary points of the Brillouin zone [Fig.1.b.]. We give here some elements of a molecular analysis of this local order [\$ 1] and non equilibrium behaviour [ 5 2]. The $X-r a y$ measurements were performed on an automatic diffractometer in experimental conditions which have been described previously $/ 6 /$.

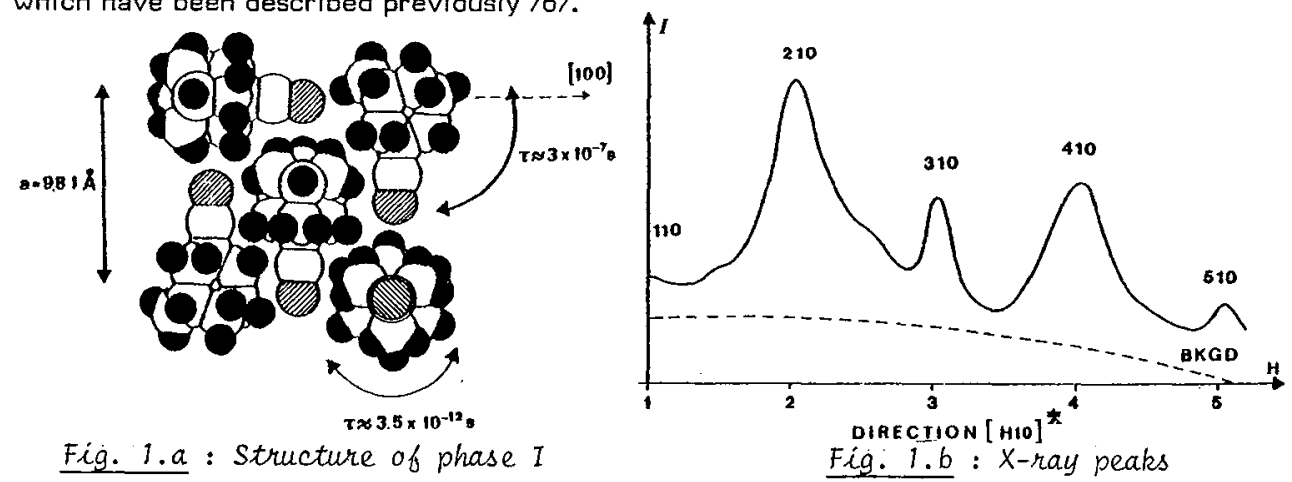


1. Local order - The presence of spots at the $\times$ points shows an antiparallel ordering of the molecules. This order develops along the $\langle 100\rangle$ axis with a local doubling of the lattice parameter. The minimum symmetry break corresponds to a local tetragonal order. To preserve the overall cubic symmetry. 3 possible orientations of domains [modulo $\Pi$ ] and 3 corresponding sets of spots are implied. For one of them we have collected 37 independent superstructure reflections. Due to annealing effects [\$ 2], the data have been collected after an annealing of 5 days at $160 \mathrm{~K}$. The superstructure reflections are far less intense than the main Bragg peaks and their broadening shows that the order is spacially very limited -In the simplest approximation of perfectly ordered domains of the same size. the Scherrer formula gives a domain diameter of about 4 unit cells $[40 \AA]$ - Nevertheless a sizable modulation of the peak intensities is observed : it is enough to discriminate between several possible models for ordered microstructures thanks to structure factor calculations.

There are two types of molecular rotations but it is clear that an ordering with respect to the axial rotations requires a previous ordering of the dipolar axes. So we have first tested this effect on the intensities. Considering the 6 possible equilibrium orientations of the dipoles, two local structures can be imagined. They differ in the arrangement of the dipoles with respect to the 4 -fold axis chosen as tetragonal axis for the domain.

[1] Dipoles are aligned in planes perpendicular to the tetragonal axis. The orientations are reversed from one plane to the following one and opposite orientations are related by a glide mirror. This structure is orthorombic Cmcm. Each tetragonal direction gives rise to two orthorombic domains of this kind [modulo II] with dipoles at $90^{\circ}$ from each other [Fig. 2). These domains diffract incoherently and the conjonction of their own extinction rules leads, for example for the $\vec{c}$ tetragonal axis, to a systematic extinction for [0 $0 I_{C}$ ] with $\mathrm{I}_{\mathrm{C}}$ being odd.

\section{Fig. 2 : Orthorambic dipolar order: model (1).}

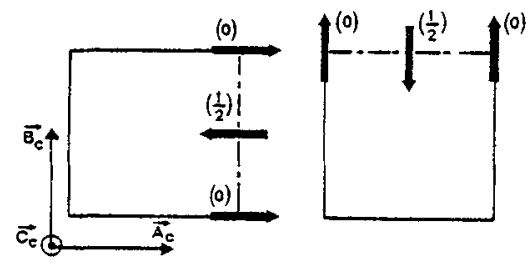

[2] The other possible structure is really tetragonal $P 4 / \pi \mathrm{mm}$. The dipoles are parallel to the tetragonal axis. In each cell there are molecules in opposite orientations related by a glide mirror perpendicular to the 4 -fold axis. This leads to the extinction for $\left[h_{c}, k_{c}, o\right]$ with $h_{c}$ and $k_{c}$ being odd [c tetragonal axis][Fig. 3.a].

We have performed a preliminary analysis by comparing the observed and calculated intensities. The latter were calculated from the atomic positions and temperature factors for the average structure $/ 7 /$. assuming a free axial rotation. The collection of data shows that, all the $X$ point reflections appear with more or less intensity. This leaves out the two models described above in their ideality. Model [1] is in complete disagreement with the data: theoretically extinguished spots in 003, 005 and 007 appear with a strong intensity. The model attributes very strong intensities to peaks of the $h_{c} k_{c} \circ\left[h_{c}+k_{c}=2 n+1\right]$ type which are in fact rather weak. Model [2] reproduces on the whole the experimental features but the predicted extinntions are not observed.

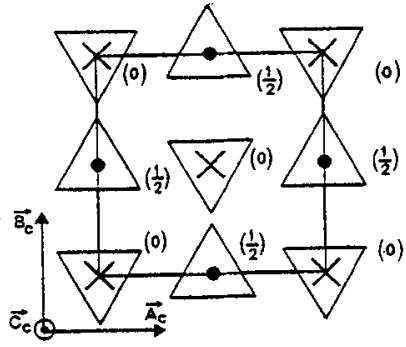

Fig. 3.6 : Resulting axial correlations of steric origin.

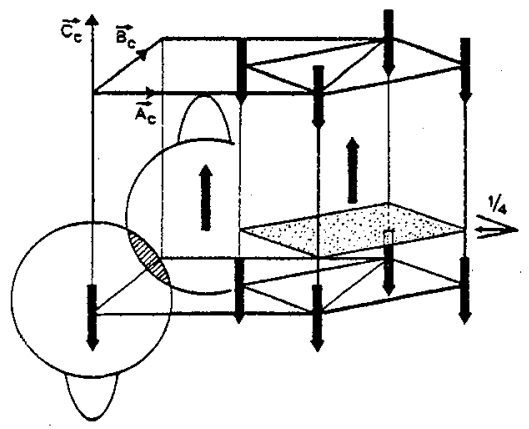

Fig. 3.a : Tetragonal dipolar order: (model 2). 
We managed to improve model [2] by ruling out the hypothesis of a free axial rotation. We considered a model with 4 axial equilibrium positions compatible with the results of the structure $/ 7 \%$ In order to suppress the extinction effect of the glide mirror, the adamantyl groups -symbolized by a triangle- must locally order themselves in the antiparallel way as shown in Fig. 3.b. This gives correct intensities for the scattering at 310 and 510 .

The antiparallel ordering of dipoles as described by model [2] is the most likely arrangement to minimize the intermolecular potential when the underlying cubic lattice is imposed. The real minimum could thus involve a slight tetragonal deformation. This conjecture is supported by the observed volume contraction to be discussed below. This ordering imposes the meeting of bulky adamantyl groups. As shown in Fig. 3.a. this occurs between neighbouring molecules of two following planes for which the dipoles do not point towards each other. The observed axial ordering likely results from correlations of steric origin between some axial configurations. This effect which is enhanced by the volume contraction is to be connected with the broad distribution of axial relaxation times recently observed under $\mathrm{Tg}$ [Amoureux et al. to be published]. To conclude, it must be pointed out that there is no connection between the orientational short range order described above and the long range order of the monoclinic phase which is stable at the same temperature /6.3/. In the latter,the dipoles are located along the 3 -fold axis of a pseudo cubic cell. This involves an important deformation of the lattice. It certainly results in a high energy at the interface with the cubic phase - which explains the important abserved undercooling.

\section{Non equilibrium behaviour}

- Kinetics of ordering. When measured at a certain temperature under Tg, the intensity and width of the superstructure peaks strongly depend on the quenching rate. After a rapid quenching. this non equilibrium behaviour is revealed by a slow growing of peaks during an isothermal annealing. The curve of Fig. 4 shows the isothermal variation of the 121 peak intensity at $156 \mathrm{~K}$. These kinetics gradually slow down but an equilibrium could not be really approached after a $B$ days annealing. We have no information on the first 30 minutes of the evolution but we can estimate a minimum relaxation time - at the beginning of the process. as it appears on Fig. 4 - to be of $\tau_{p} \approx 40$ hours. The determination of the HWHM of the profile, therefore of the characteristic length $\xi$ of the domains is less precise. But several experiments, at different temperatures on different samples lead us to observe that the peak intensity grows at least like $\xi^{3}$ - more rapidly than had been previously suspected $/ 6 /$. Let us mention that equivalent peaks corresponding to the different kinds of domains follow equivalent evolutions and therefore preserve the overall cubic symmetry.

Fig. 4: Isothermal $(156 \mathrm{~K})$ relaxation of the intensity after quenching.

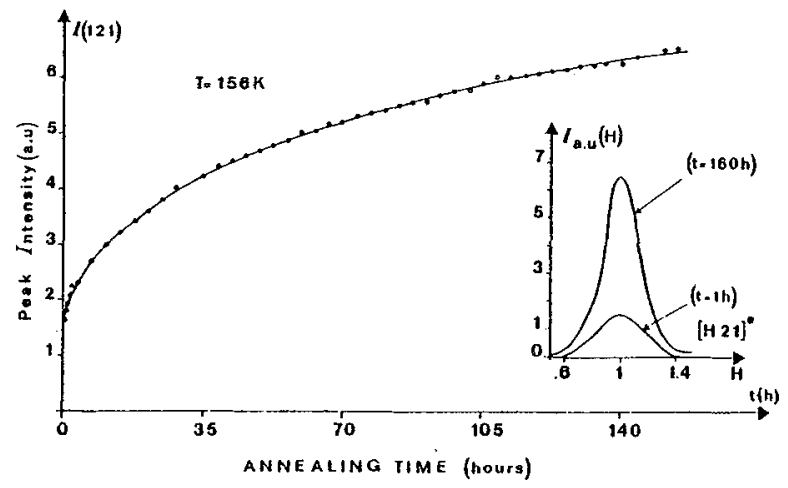

- Volume relaxation. The simultaneously repeated collection of some main X-ray Bragg reflections revealed a noticeable contraction of the cubic parameter $a$. The curve of Fig.5 shows the isothermal variation of a as measured from the set of the 24 equivalent 511 reflections. An annealing of $B$ days at $156 \mathrm{~K}$ leads to the ultimate value $\Delta \mathbf{a} / \mathbf{a}=1.310^{-3}$. Let us note that the corresponding change of the unit cell volume $\Delta v / v=410^{-3}$ is of the same order as the volume jump when ordering at the first-order transition between the stable cubic and monoclinic phases where one has $\Delta v / V=5 \quad 10^{-3} / 3 /$. A lawer limit of the relaxed cubic parameter a can reasonably be taken as the cubic root of the monoclinic unit cell volume taken at the same temperature. The time dependence of Fig. 5 thus fits an exponential law with the characteristic time $\tau_{a} \simeq 45$ hours. 
Fig. 5 : Isothermal (156 K) relaxation of the parameter after quenching.

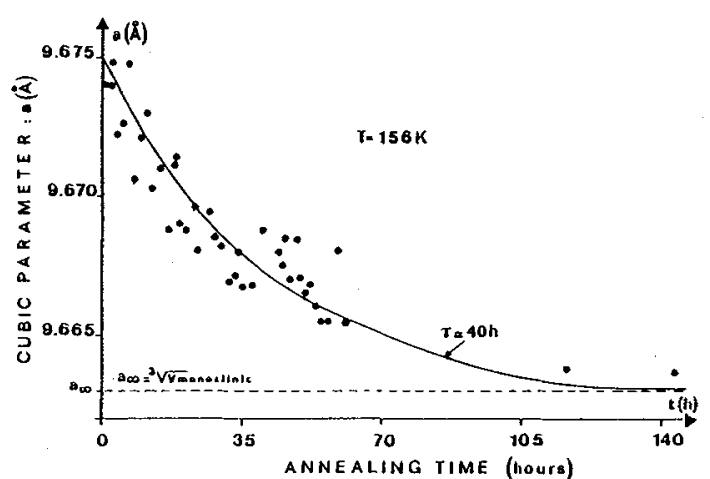

- Discussion - Time scale of the molecular reorientations-The preceeding results show that the orientationally disordered CN-ADM quenched below Tg relaxes towards a metastable equilibrium state. This relaxation is not the kinetic effect of crystallization into the most stable monoclinic phase. If the limited local ordering in the quenched phase were described by an $O-Z$ homogeneous correlation function, the local order to which the later gives rise would induce a peak intensity growing like $\xi^{2}$. An evolution like $\xi^{3}$ at least. better evokes an ordering at localized regions. We shall examine this question further in a forthcoming paper. But one possibility is that of nucleation and [or] growth of antiferro ordered domains in an orientationally disordered matrix.

These kinetic effects require thermally activated reorientations of the dipolar axis as outlined on Fig. 6. Since there is perfect fitting of the reciprocal lattices, this ordering takes place coherently with the matrix. The observed volume contraction would thus correspond to slight local tetragonal deformations of the lattice. It results from a translational coupling with the orientational ordering. The similarity and simultaneity of both kinetics show that contraction and ordering are correlated in some way.

Fig. 6: Possible molecular process for the displacement of a domain boundary $(---)$. Reorientations of the dipolar axis are required. Let us note that the interface is coherent.

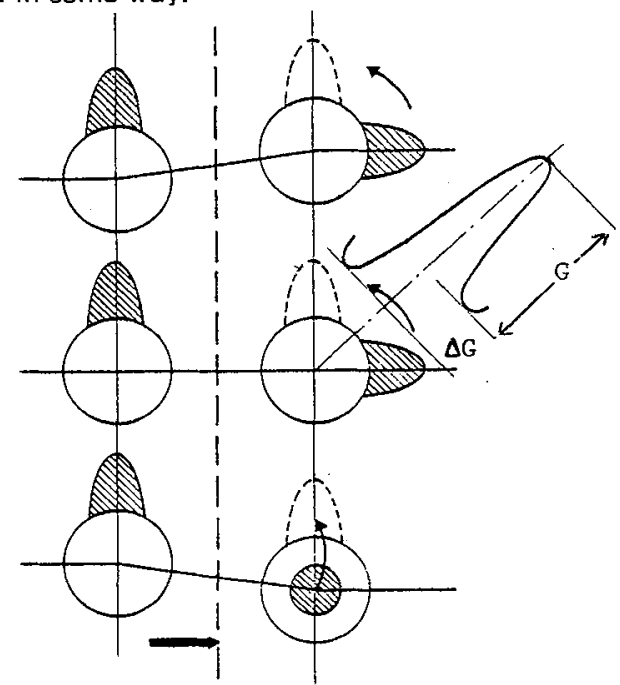

According to the process outlined in Fig. 6. We can roughly estimate the domain interface velocity $V$ in terms of the molecular relaxations involved in the ordering. Let $\tau$ be the time scale of residence of a molecule in one of the six orientations of the disordered phase at temperature $T$. It is a non collective parameter determined by the height of the potential barrier $G$ dividing two orientations $\left[\tau=\tau_{0} \exp G / k T\right]$. Due to the symmetry. if we start from a given orientation. we can reach four different orientations by a single jump. Let $\Delta G$ be the difference in free energy that drives the molecules of the boundary towards the equilibrium orientation of the domain. The rate equation far the reorientational transfert gives:

$$
V \simeq(4 a / \tau)[1-\exp (-\Delta G / k T)]<a / \tau
$$


[a = lattice parameter ]. Since the increase of correlation length between the first hour and the end of the anneal is about $\Delta \xi=a$, the observed velocities are less than $a / \tau_{\exp }\left[\tau_{\exp } \simeq 40\right.$ hours at $156 \mathrm{KJ}$. The experimental time scale of the kinetics is thus a maximum value of the non collective molecular relaxation time. Dielectric susceptibilities have been measured in plastic and undercooled plastic phase in the range $235-350 \mathrm{~K} / 9.10 /$ and around $185 \mathrm{~K} / 9 /$. The overall evolution of the frequency of the maximum absorption $f_{d}$ has been described by a Vagel-Fulcher [V.F] law. Its extrapolation down to the annealing temperatures of the present work gives values of relaxation times $\left[\tau_{d}=1 / 2 \Pi f_{d}\right]$ which are orders of magnitude higher than the above $\tau_{\text {exp }}$ value [Fig. 7 ]. At $156 \mathrm{~K}$ the relaxation time becomes 15500 hours for the fit of $/ 9 /$ and 985 hours for the fit of $/ 10 \%$. Of course we can not expect the identity of the relaxation times but such a difference is intriguing. More experiments are needed to understand this apparent change from a V.F behaviour to a pseudo Arrhenius one.

Fig. 7 : Arrhenius plot of relaxation times:

- From the present kinetics experiments at Low temperature;

- : extrapolation of the dielectric experiments of Amoureux et al. 19/;

-.-: extrapolation of the dielectric experiments of Johari et al. $/ 10 \%$.

References

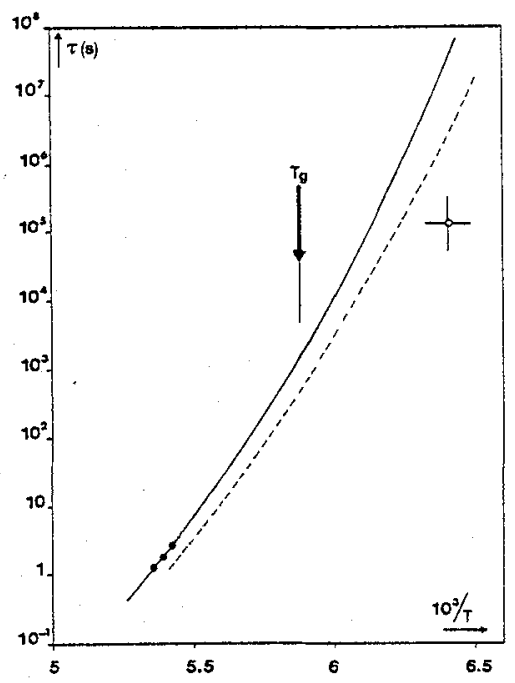

/1/ H. Suga (1983), Pure and Appl. Chem., vol. $55 \mathrm{n}^{\circ} 3,427-436$, G.P. Johari (1976), Ann. N.Y. Acad. Sci. 279, 101.

/2/ M. Foulon, J.P. Amoureux, J.L. Sauvajol, J. Lefebvre, M. Descamps (1983), J. Phys. C., 16, L 265.

/3/ M. Foulon, J.P. Amoureux, J.L. Sauvajol, J.P. Cavrot, M. Muller (1984), J. Phys. C. $17,4213-4229$.

/4/ R. Vacher et al. (1983), xVth Eur-Symp-on Dynamical properties of Solids LEUVEN.

/5/ M. Descamps, C. Caucheteux, G. Odou, J.x. Sauvajol (1984), J. Phys. Lett. 45 , L $719-727$.

/6/ M. Descamps, G. Odou, C. Caucheteux (1985), J. de Phys. Lett. 46, L261-L265.

/7/ M. Foulon, J. Lefebvre, J.P. Amoureux, M. Muller, D. Magnier, J. de Phys. (1985).

19/ J.P. Amoureux, G. Noyel, M. Foulon, M. Bée, L. Jorat (1984), Mol. Phys. 52, 1, 161-171.

/10/ K. Pathmanathan, G.P. Johari, to be published J. Phys. C. 